An automatic system for the wiring of Drift Chambers for modern high intensity and high precision particle physics experiments

To cite this article: G. Chiarello et al 2020 JINST 15 C07034

View the article online for updates and enhancements.

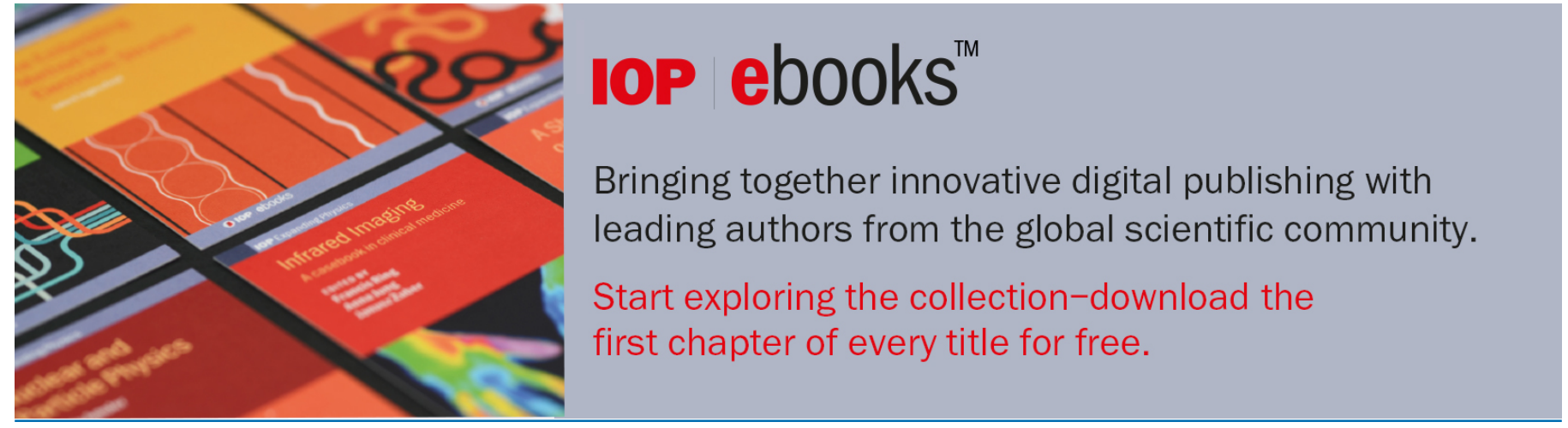




\section{An automatic system for the wiring of Drift Chambers for modern high intensity and high precision particle physics experiments}

G. Chiarello, ${ }^{a, b, 1}$ A. M. Baldini, ${ }^{e}$ G. Cavoto, ${ }^{a, b}$ F. Cei,,${ }^{e, f}$ M. Chiappini, ${ }^{e}$ A. Corvaglia, ${ }^{d}$ M. Francesconi, ${ }^{e}, f$ L. Galli, ${ }^{e}$ F. Grancagnolo, ${ }^{d}$ M. Grassi, ${ }^{e}$ M. Hildebrandt, ${ }^{g}$ M. Meucci, ${ }^{a}, b$ A. Miccoli, ${ }^{d}$ D. Nicoló, ${ }^{e, f}$ A. Papa, ${ }^{e, f, g}$ M. Panareo, ${ }^{c, d}$ F. Raffaelli, ${ }^{e}$ F. Renga, ${ }^{b}$ G. Signorelli, ${ }^{e}$ G.F. Tassielli ${ }^{d, 1}$ and C. Voena ${ }^{b}$

a Dipartimento di Fisica dell'Università "Sapienza" di Roma,

Piazzale A. Moro 2, 00185, Roma, Italy

${ }^{b}$ INFN Sezione di Roma,

Piazzale A. Moro 2, 00185, Roma, Italy

${ }^{c}$ Dipartimento di Matematica e Fisica dell'Università del Salento,

Via per Arnesano, 73100, Lecce, Italy

'IINFN Sezione di Lecce,

Via per Arnesano, 73100, Lecce, Italy

e INFN Sezione di Pisa,

Largo B. Pontecorvo 3, 56127, Pisa, Italy

${ }^{f}$ Dipartimento di Fisica dell'Università di Pisa,

Largo B. Pontecorvo 3, 56127, Pisa, Italy

${ }^{g}$ Paul Scherrer Institut PSI,

5232, Villigen, Switzerland

E-mail: gianluigi.chiarello@roma1.infn.it

ABSTRACT: Tracking systems for modern particle physics experiments, both for the search of extremely rare processes, which require high momentum resolutions in ranges dominated by multiple scattering, and for the next generation of high luminosity lepton colliders, which require large active volumes and high granularity, make use of light drift chambers able to withstand high rates and, therefore, made of large numbers of closely spaced wires. For such chambers, the use of the classical feed-through technique, as wire anchoring system, cannot be implemented and it is necessary to develop an automatic system (wiring robot) to locate the wires with high accuracy and under a well monitored and uniform mechanical tension with fast and reliable operations.

Keywords: Gaseous detectors; Particle tracking detectors (Gaseous detectors)

${ }^{1}$ Corresponding author. 


\section{Contents}

1 Introduction 1

2 The wiring robot 2

2.1 Wiring system 3

2.2 Soldering system 3

2.3 Extraction system 5

3 The control system 6

4 Conclusions $\quad 6$

\section{Introduction}

Over the last half century one of the most engaging aspects of modern particle physics experiments has been the search for the extremely rare processes such as charged Lepton Flavour Violation processes (cLFV). Current experiments, like MEG [1-3] at PSI, Mu2e [4] at Fermilab and COMET [5] at J-PARC require for this purpose tracking detectors with high precision and able to withstand high rates. A typical tracking detector is a full stereo, high granularity drift chamber.

The MEG II positron detector is a unique volume low-mass Cylindrical Drift CHamber (CDCH) with high granularity and full stereo wires configuration. $\mathrm{CDCH}$ is composed of 9 layers of drift cells at alternating stereo angles. Each layer has 192 square cells with ratio of field wires to sense wires equal to $5: 1$ to ensure the proper electrostatic configuration. In total $\mathrm{CDCH}$ is made up of 1728 sense wires, 9408 field wires and 768 guard wires $[6,8]$. The $\mathrm{CDCH}$ wire density is $\sim 12$ wires $/ \mathrm{cm}^{2}$.

Due to the high wire density, the use of the traditional feedthrough technique as wire anchoring system cannot be implemented and therefore it was necessary to develop a new wiring strategy. The novel construction technique is based on the concept of separating the gas containment function from the wire support function [7]. This scheme eliminates the use of wire feedthroughs and imposes to use a different wire anchoring system. In addition, the developed feedthrough-less wiring technique allows for closer wire spacing, i.e. for smaller drift cells and finer chamber granularity. Moreover, it allows to use thinner and lighter field wires. Small cells and large number of wires, however, require assembly procedures with high precision which call for a novel approach to the wiring problem. For the MEG II CDCH, the gas containment function is performed by plastic spacers and by the wire Printed Circuit Boards (PCB), on which wires are soldered. The use of wire-PCB allows for higher densities of wires, accurately positioned and strung at the correct mechanical tension. The stringent requirements on the precision of wire position and on the uniformity of the wire mechanical tension impose the use of an automatic system (Wiring Robot) to operate the wiring procedures.

The wiring robot has been designed and build: 
- to wind variable pitches and variable stereo angle configuration with continuity;

- to apply a pre-defined mechanical tension to the wires and to maintain it constant and uniform through the whole chamber;

- to monitor the wire positions and their alignments within a few tens of $\mu \mathrm{m}$;

- to anchor the wires to their support with a contact-less soldering system;

- to monitor the solder quality of the wire to the supporting PCB.

\section{The wiring robot}

The wiring robot (figure 1) [7], designed and built at the INFN Lecce and University of Salento laboratories, consists of:

- WIRING SYSTEM: a semiautomatic wiring machine with a high precision on wire mechanical tensioning $(<0.05 \mathrm{~g})$ and on wire positioning $(<20 \mu \mathrm{m})$ for a simultaneous wiring of multi-wire frames;

- SOLDERING SYSTEM: a contact-less infrared laser soldering tool for anchoring the wires to the supporting PCB;

- EXTRACTION SYSTEM: an automatic handling system for removing the multi-wire frames from the wiring system and for storing them.

All subsystems of the wiring robot are managed and synchronized with a real-time system, based on a National Instrument Compact RIO platform ${ }^{\circledR}$ [9]. The wiring robot has been used for the wiring phase of the MEG II CDCH.

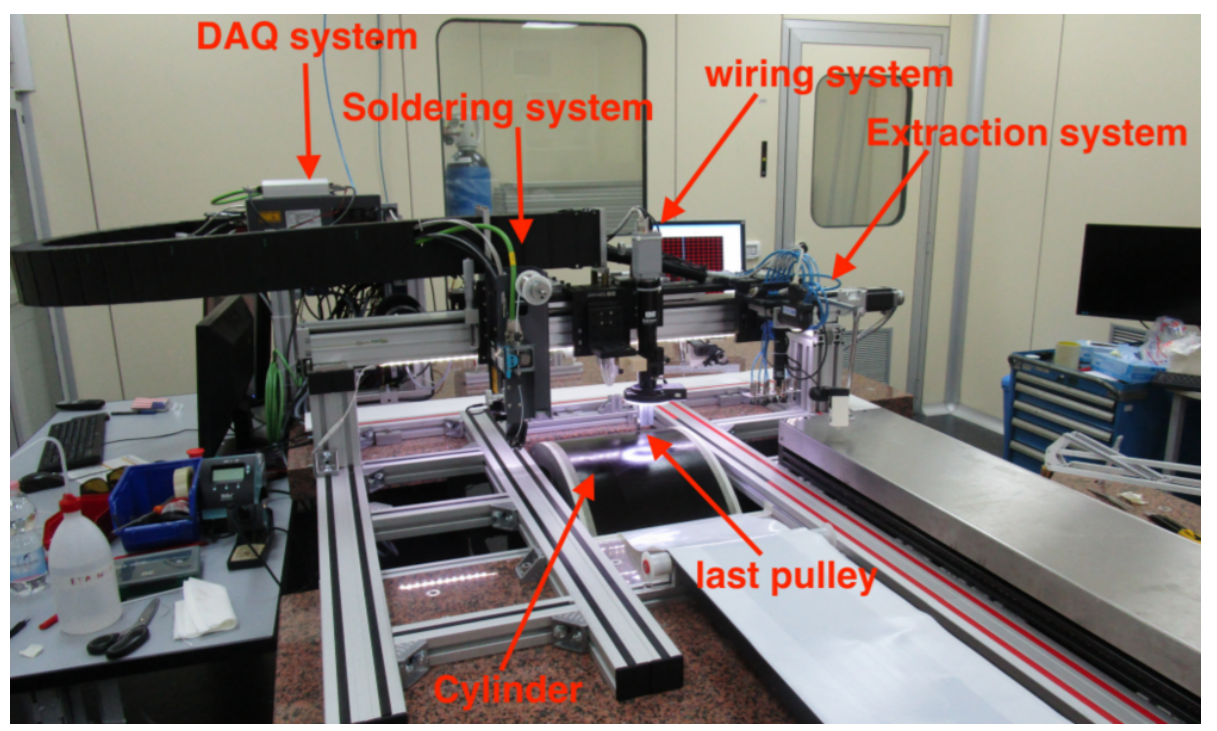

Figure 1. The wiring robot. 


\subsection{Wiring system}

The task of the wiring system is to distribute the wire along a helicoidal trajectory with high precision and with a constant pre-defined mechanical tension. The system winds a multi-wire layer made up of 32 parallel wires at any stereo angle (figure 2: right). In order to obtain a multi-wire layer, the wire-PCB is positioned and aligned in a template on the winding cylinder. The wiring system is composed of different parts:

- a wiring cylinder with a radius of $33 \mathrm{~cm}$, covered with a thin rubber layer to avoid wire slippage. The diameter of the cylinder is related to the length of the MEG II CDCH;

- an electromagnetic brake applied to the wire spool and controlled by a strain gauge for feedback on the correct wire mechanical tension;

- a system of pulleys to drive the wire.

A multi-wire layer, composed of 32 parallel wires, is formed in a single operation by winding the wire along a helix, in order to intersect it over the wire-PCB pads with the desired pitch. The pitch is made by a system of synchronized stepper motors through the Compact RIO system and controlled by a digital camera with a software developed with LabVIEW® [10]. The position of the wire is controlled during the wiring phase by a digital camera and a linear slide. The accuracy on the position is $<20 \mu \mathrm{m}$ (figure 2: left). The wire mechanical tension is set to a nominal value and it is monitored by a high precision strain gauge and corrected with a real-time feedback system, acting on the wire spool electromagnetic brake.

The wire tension variations are of the order of $\pm 1.5 \mathrm{~g}$, without the feedback system, because of the mechanical tolerances. The feedback system reduces these variations to less than $\pm 0.05 \mathrm{~g}$ (figure 3).
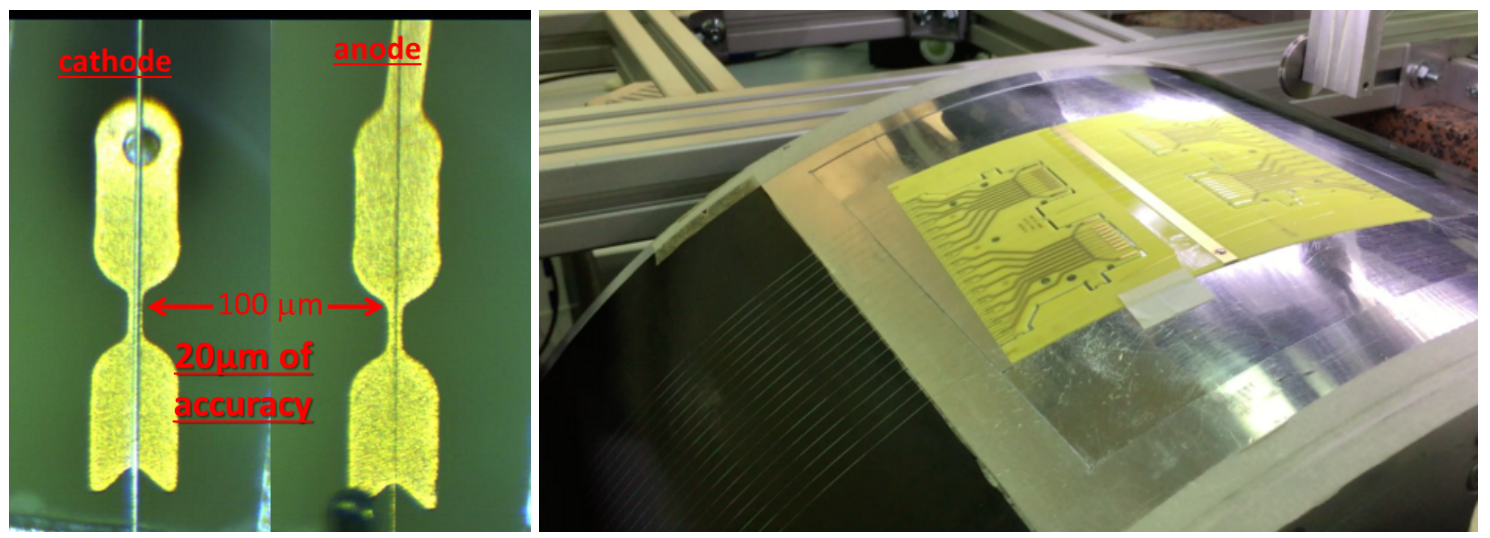

Figure 2. Left: position of the wire on the pad of the wire-PCB. Right: multi-wire plane wired.

\subsection{Soldering system}

The soldering phase is accomplished by an IR laser soldering system (LASCON Hybrid [11]) consisting of a solder wire feeder, a $68 \mathrm{~W}$ IR laser, a pyrometer and a digital camera, as shown in figure 4 . The digital camera allows to see in real time the soldering operations and the pyrometer 

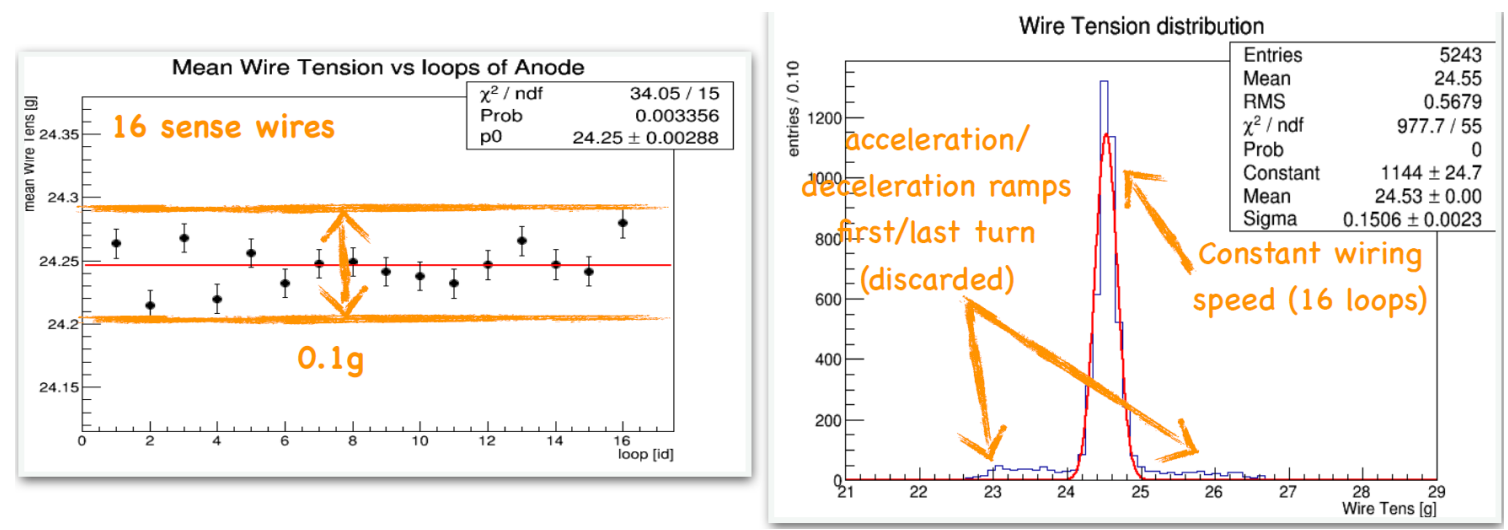

Figure 3. Left: average anode wire tension for each loop. Right: the distribution of the anode wire tension during the winding.

monitors the temperature at the laser focus. Each wire is fixed at both ends while still constrained around the winding cylinder under its own tension. The laser system is controlled by the Compact RIO and it is synchronized with the positioning system to localize the soldering pad, by using a pattern matching software developed with LabVIEW ${ }^{\circledR}[10]$. All the soldering parameters (temperature, soldering time, solder wire length and feeding speed) are defined in order not to cause any damages to the wires, through a proper script embedded in LabVIEW® ${ }^{\circledR}[10]$. In figure 5 the variation of the parameters during the soldering phase are shown. To avoid damages to the very frail $\mathrm{Al}$ field wires, the soldering is done at a temperature of $\sim 180^{\circ} \mathrm{C}$ by using a low melting point $\left(\sim 160^{\circ} \mathrm{C}\right)$ tin alloy. ${ }^{1}$

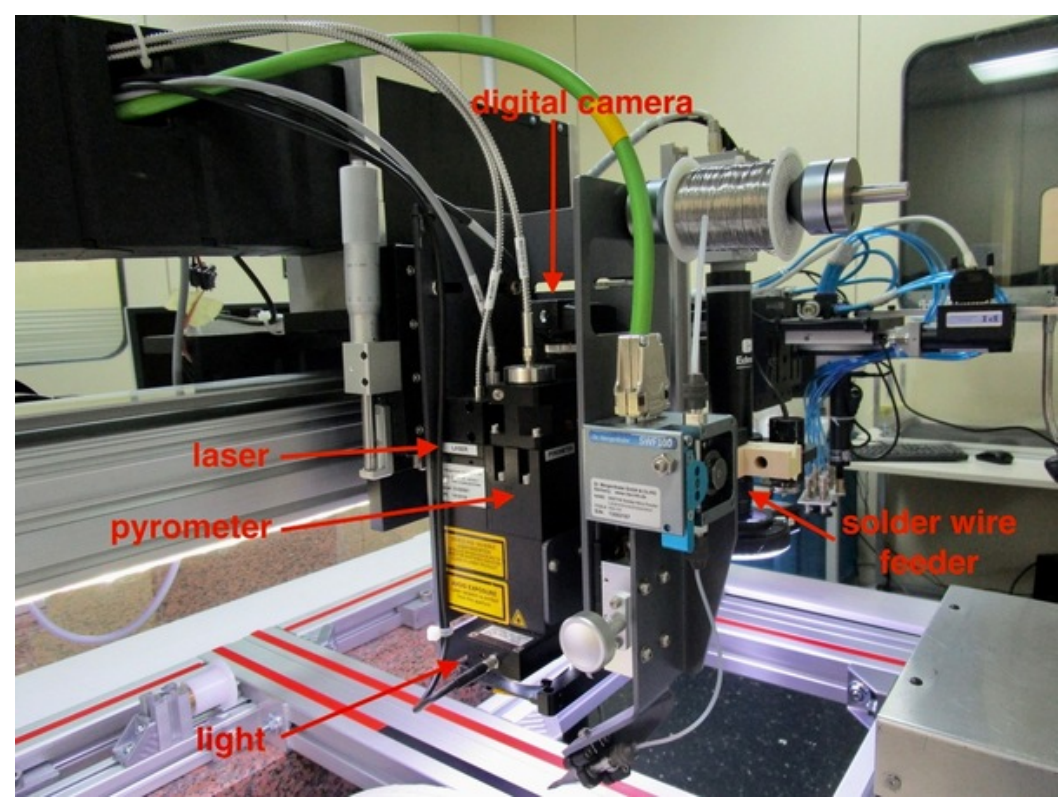

Figure 4. The Laser system.

${ }^{1}$ https://www.kester.com/products/product/245-flux-cored-wire. 

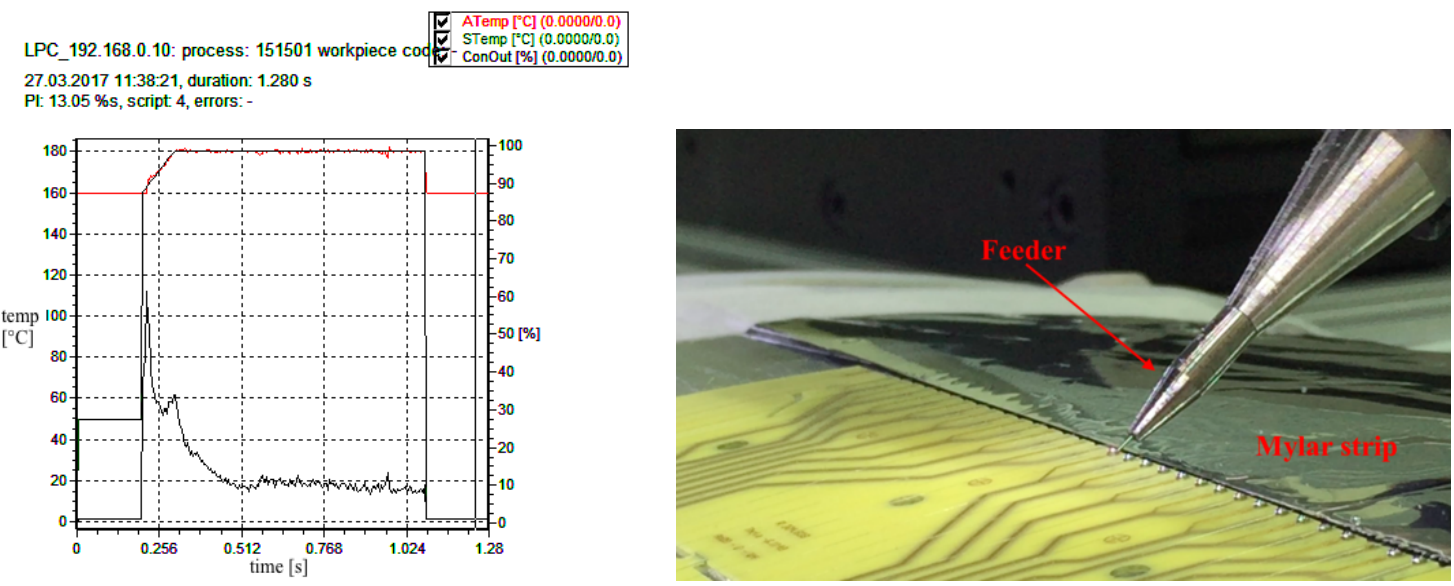

Figure 5. Left: the laser spot temperature (green line), the pyrometer value (red line) and the control output (black line) of the laser during a soldering. Right: soldering Phase (a mylar strip is used to protect the threads in this phase).

\subsection{Extraction system}

The layer of soldered wires, wounded around the cylinder must be unrolled and de-tensioned for storage and transport. This is accomplished with the extraction system (figure 6: left). After removing the excess wire, the first wire-PCB (figure 6: right) is lifted off from the cylinder surface with a linear actuator connected to a set of vacuum-operated suction cups and placed on the storage and transport frame. The unrolling is accomplished by synchronizing the cylinder rotation with the linear displacement of the frame. Once the layer of soldered wires is completely unrolled, the second wire-PCB is lifted off from the cylinder, as the first one, and placed on the frame. The frame hosts two supports made of poly-carbonate, dedicated to hold the wire-PCBs at the correct position by means of nylon screws. One of the two supports can slide into the frame to adjust the wire length by means of a longitudinal threaded rod.

After the extraction phase, the multi-wire frames are examined and stored in sets of 13 (for a full layer of 12 sectors plus one spare). The 3 sets of 13 multi-wire frames, wrapped in a welded seal bag to avoid contamination from outside and equipped with valves in order to be flushed with dry gas, are then shipped to the assembly site in Pisa.
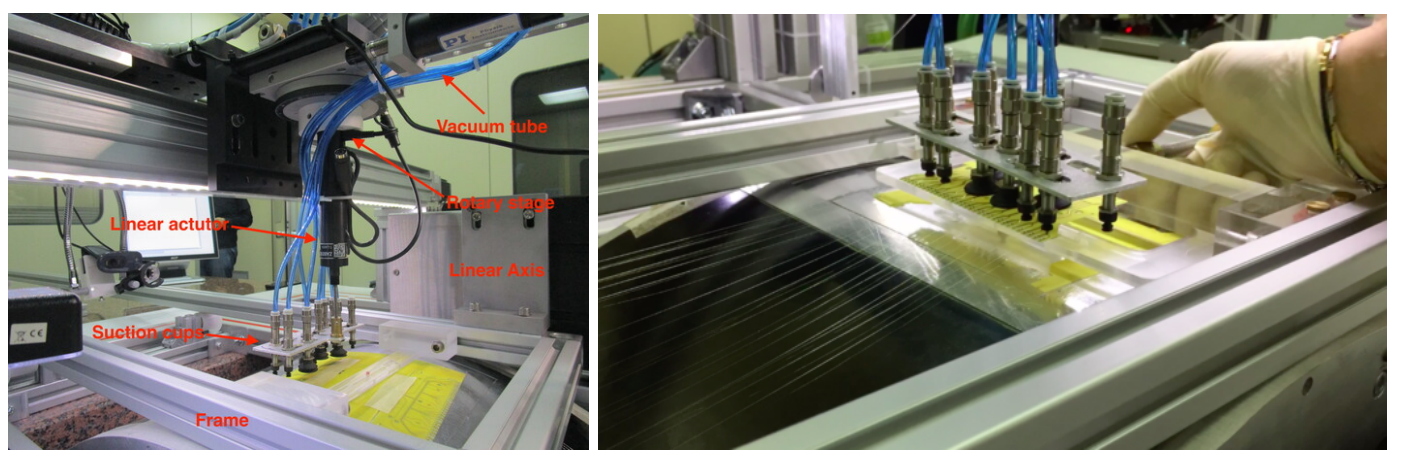

Figure 6. Left: extraction system. Right: wire PCB is lifted off from the cylinder. 


\section{The control system}

The Control system must monitor and accurately synchronize all the systems described in the previous sections. The hardware of the control system is the Real-Time PowerPC Controller NI cRIO-9024®and the chassis NI cRIO-9112® [9]. The chassis allows the parallel communication of several additional modules for the control of the sensors and the devices of the wiring robot.

The control system software is entirely developed in LabVIEW ${ }^{\circledR}[10]$ to perform the correct synchronization of the various phases. Figure 7 shows the main screen of the software for the management of the movements determined by the operator and the different steps for winding a multi-wire plane.

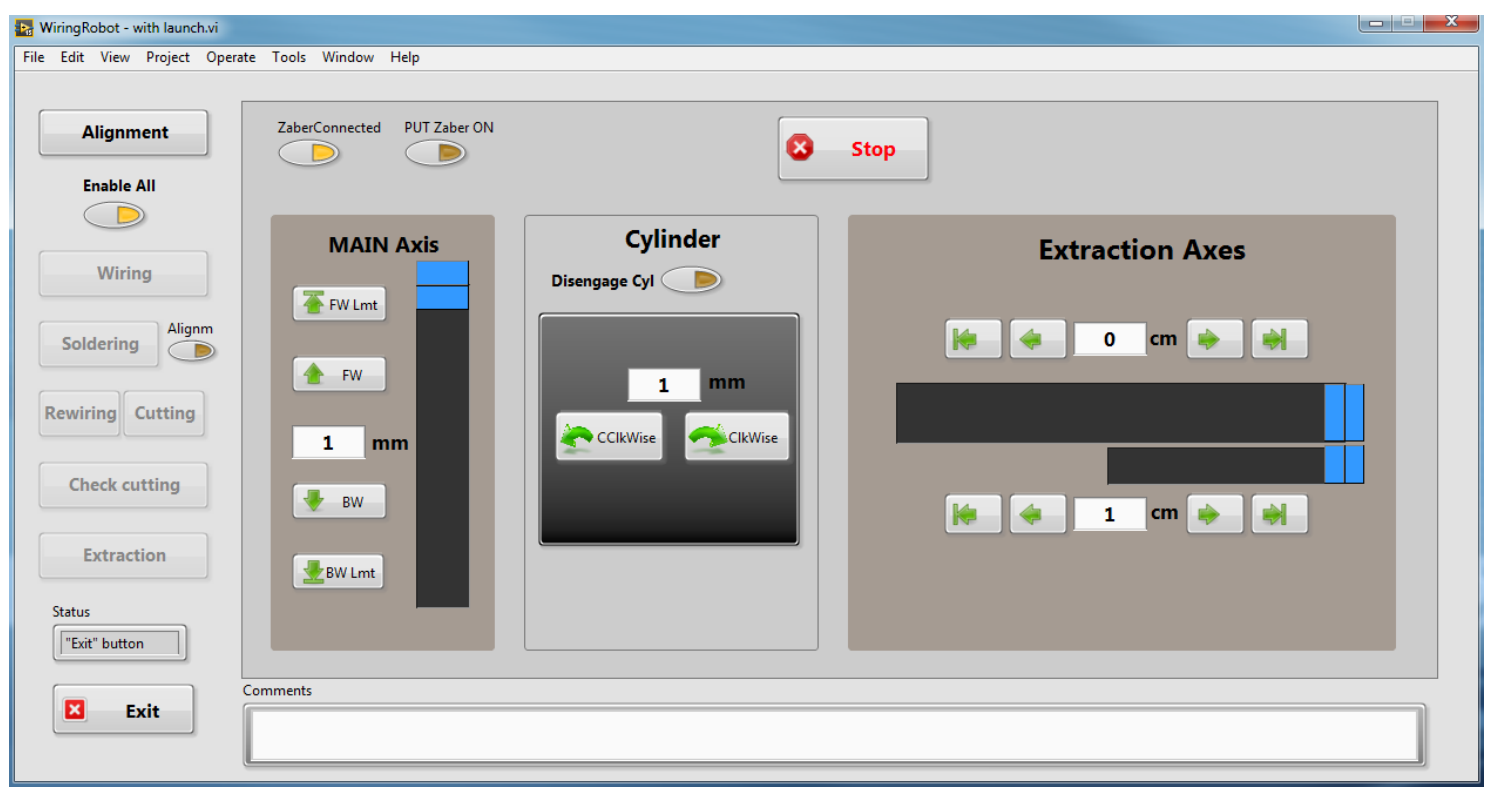

Figure 7. The main screen of the software.

\section{Conclusions}

A novel technique for wiring high granularity and high transparency drift chambers with high thin wires densities with a feedthrough-less strategy has been described. It makes use of an automatic system for a precise positioning and for a well controlled and uniform mechanical tension of the wires. A contact-less laser soldering system is used to solder the wires without mechanical and overheating stresses.

The wiring robot was used for the construction of the drift chamber of the MEG II experiment at PSI.

\section{References}

[1] MEG collaboration, New limit on the lepton-flavour violating decay $\mu^{+} \rightarrow e^{+} \gamma$, Phys. Rev. Lett. 107 (2011) 171801 [arXiv: 1107 .5547]. 
[2] A.M. Baldini et al., MEG Upgrade Proposal, arXiv: 1301.7225.

[3] MEG II collaboration, The design of the MEG II experiment, Eur. Phys. J. C 78 (2018) 380 [arXiv: 1801.04688].

[4] Mu2e experiment, http://mu2e.fnal.gov.

[5] COMET experiment, http://comet.kek.jp.

[6] A.M. Baldini et al, The ultra light Drift Chamber of the MEG II experiment, Nucl. Instrum. Meth. A $958(2020) 162152$.

[7] G. Chiarello, The tracking detector of the MEG II experiment, Ph.D. Thesis, University of Salento (July 2017), https://meg.web.psi.ch/docs/theses/chiarello_PhD.pdf.

[8] A.M. Baldini et al., Single-hit resolution measurement with MEG II drift chamber prototypes, 2016 JINST 11 P07011 [arXiv: 1605 . 07970].

[9] National Instrument, The CompactRIO Platform - National Instruments, http://www.ni.com/compactrio/.

[10] National Instrument, LabVIEW System Design Software, http://www.ni.com/labview.

[11] Dr. Mergenthaler GmbH \& Co. KG, LASCON Hybrid System, https://ma-info.de/en/processing-heads.html. 